\title{
Bilateral Sudden Sensorineural Hearing Loss (SSNHL) Following Lumbar Puncture: A Case Report
}

\author{
Bezas V*, Hammersen JJ, Issing PR \\ Department of Otorhinolaryngology, Head-Neck and Facial Plastic Surgery, Klinikum Bad Hersfeld, \\ Germany
}

\begin{abstract}
Sudden sensorineural hearing loss affects frequently one ear (unilateral). A bilateral sudden SNHL is the rare form of SSNHL ( $5 \%$ of all cases) having mostly as aetiology diseases with a specific clinical pathology. Many case reports of a bilateral SSNHL in the literature include not only adult populations but also paediatric cases. A bilateral SSNHL following a lumbar puncture belongs to the rarest iatrogenic complications and it is an uncommon aetiology causing transient or permanent unilateral or bilateral hearing impairments. We report a case of a 83-year-old woman who developed on her chronic hearing loss a bilateral SSNHL after lumbar puncture and we discuss this case reviewing the current literature.
\end{abstract}

\section{Introduction}

According to the definition of the National Institute on Deafness and other Communication Disorders, a sudden sensorineural hearing loss (SSNHL) is a sudden hearing impairment with a rapid manifestation in one or both ears within three days and a minimum of $30 \mathrm{~dB}$ hearing loss or more, over 3 consecutive frequencies in the patient's audiogram [1]. In the US about 66,000 new cases of SSNHL are referred annually [2]. Between 2014 and 2016 15, 9\% of the U.S. adults over 18 years developed any hearing loss [3]. SSNHL in children ( $<18$ years) is rare with an incidence of 6 , $6 \%$ published for younger as 18 years old patients [4].

Although a bilateral SSNHL is not the predominant form of a SSNHL, it is mainly associated with systemic pathologies such as infections, ototoxicity, vascular, neoplastic, and autoimmune diseases. Besides these causes, we have to mention iatrogenic aetiologies such as microembolic surgical complications as well as hemodynamic or ototoxic complications after anaesthesia associated to cardiopulmonary, orthopedic or urologic surgeries. The sudden-onset hearing loss post spine surgery was described as a rare disastrous complication, resulting in deafness to the patients [5]. Unilateral permanent sudden sensorineural hearing loss after spinal- anesthesia for caesarean section was published as case report [6]. The common factor in many of those surgical cases was spinal anaesthesia [7].

Lumbar puncture (LP) is a common and important medical procedure giving access to the cerebrospinal fluid (CSF) either as part of therapeutic / diagnostic technique with an injection of drugs or as examination tool of the CSF for a variety of infectious or non-infectious diseases. It will be performed from physicians (neurologists, neurosurgeons, emergency physicians, paediatricians and anesthesiologists). One of the rare late complications of LP is a hearing impairment.

\section{Case Report}

A female patient, 83- years old, was urgently admitted to our hospital suffering from an acute onset of a subjective bilateral hearing impairment. She complained of a rapid hearing loss after a diagnostic LP to analyse the CSF for a Lyme borreliosis 5 days ago. The recommendations of her physicians to wait for a spontaneous recovery of the hearing impairment did not improve her symptoms. A bilateral subjective chronic tinnitus, although bothersome, did not change its acoustic characteristics and loudness along the episode. Other neurological symptoms or failures were not reported. Dizziness, headaches, fever, vertigo or aural fullness were not described by the admission. According to the medical reports the LP was performed without complications and the examinations of the CSF revealed no infection.

The ENT- examination showed no infectious marks in both ears, the ear canals and the eardrums were intact and the tympanic cavities were normally ventilated. In Weber's test the sound of the tuning fork was perceived in the midline and

*Corresponding author: Bezas V, Department of Otorhinolaryngology, Head-Neck and Facial Plastic Surgery, Klinikum Bad Hersfeld, Germany

Accepted: November 05, 2021

Published online: November 08, 2021

Citation: Bezas V, Hammersen JJ, Issing PR (2021) Bilateral Sudden Sensorineural Hearing Loss (SSNHL) Following Lumbar Puncture: A Case Report. J Head Neck Surg 3(2):160-162 
Citation: Bezas V, Hammersen JJ, Issing PR (2021) Bilateral Sudden Sensorineural Hearing Loss (SSNHL) Following Lumbar Puncture: A Case Report. J Head Neck Surg 3(2):160-162

the Rinne's test was positive. The motor functions of the facial nerve were bilateral intact. There was no sign of spontaneous or provoked nystagmus using Frenzel spectacles.

The audiological assessment of the patient on admission confirmed a moderate- to- severe bilateral SSNHL (Figure 1).

The patient was commenced on systemic corticosteroids with a dose of $250 \mathrm{mg}$ prednison once daily, according to the current German guidelines in case of SSNHL, over three consecutive days. The patient was informed and gave written consent about the side effects and the possibility of success of the proposed therapy. After 3 days and without complications the patient confirmed an improvement of symptoms.

We repeat audiological assessment at the third day of the treatment to examine the quality of the hearing. The tympanometry showed a normal compliance in the middle ear both sides (type A). The pure ton audiometry revealed an improvement of the hearing corresponding to a chronic hearing loss suitable the patient's age (Figure 2).

\section{Discussion}

Although bilateral sudden sensorineural hearing loss is rarely reported in the literature, it seems to be more commonly related to diseases that have a systemic aetiology. That's why the hearing prognosis is poorer and the outcomes are not promising. In contrast to a unilateral disease entity, in bilateral sudden sensorineural hearing loss an idiopathic aetiology is rare. Younger patients are more likely to be affected on infectious, toxic or autoimmune diseases in the third decade of their life. Older patients in the fifth decade of their life suffer more often from vascular, neoplastic or iatrogenic causes when they develop a bilateral SSNHL. Among them, iatrogenic causes are often related to a recent operation or anaesthesia. In this case acute insults have sustained the bilateral SSNHL [8].
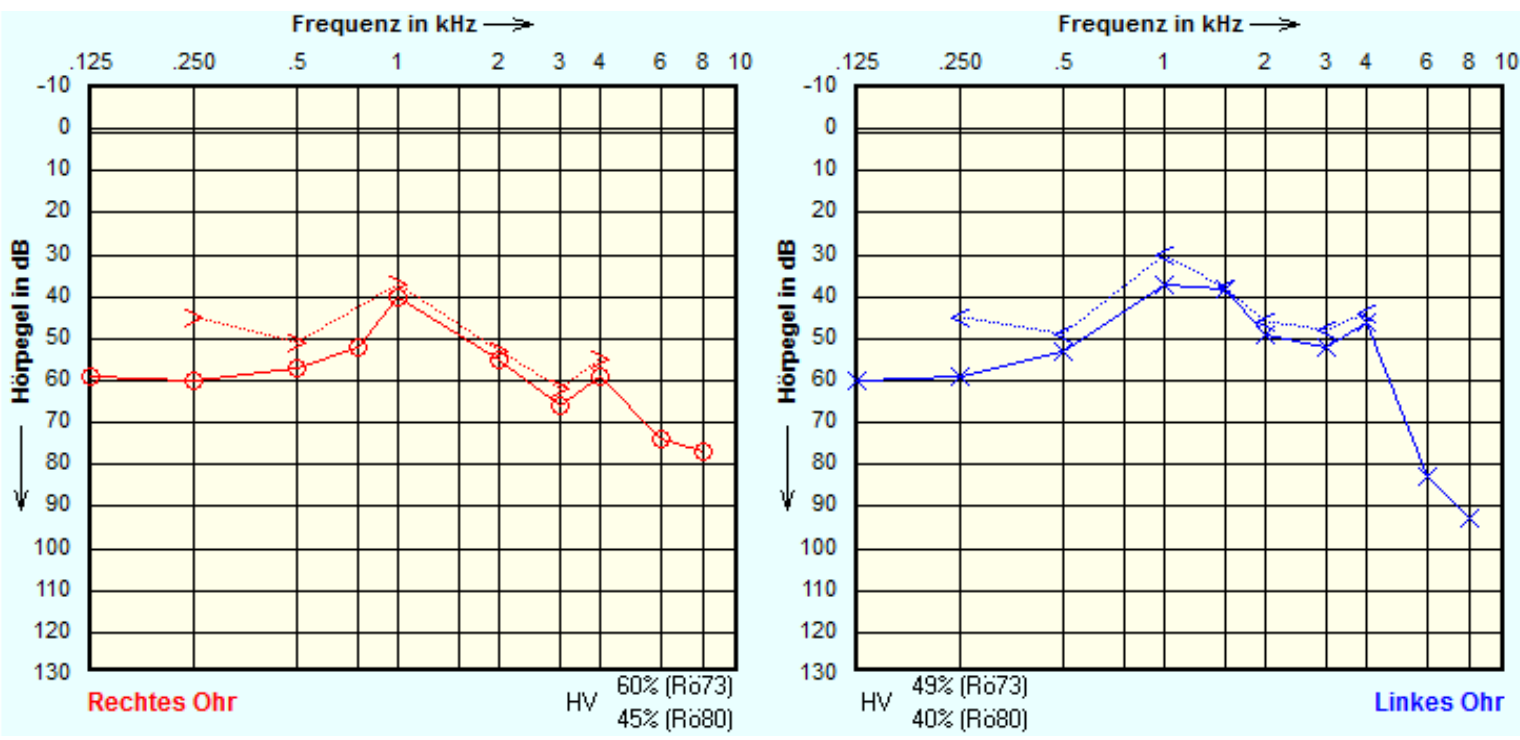

Figure 1: Initial audiogram on the day of admission.
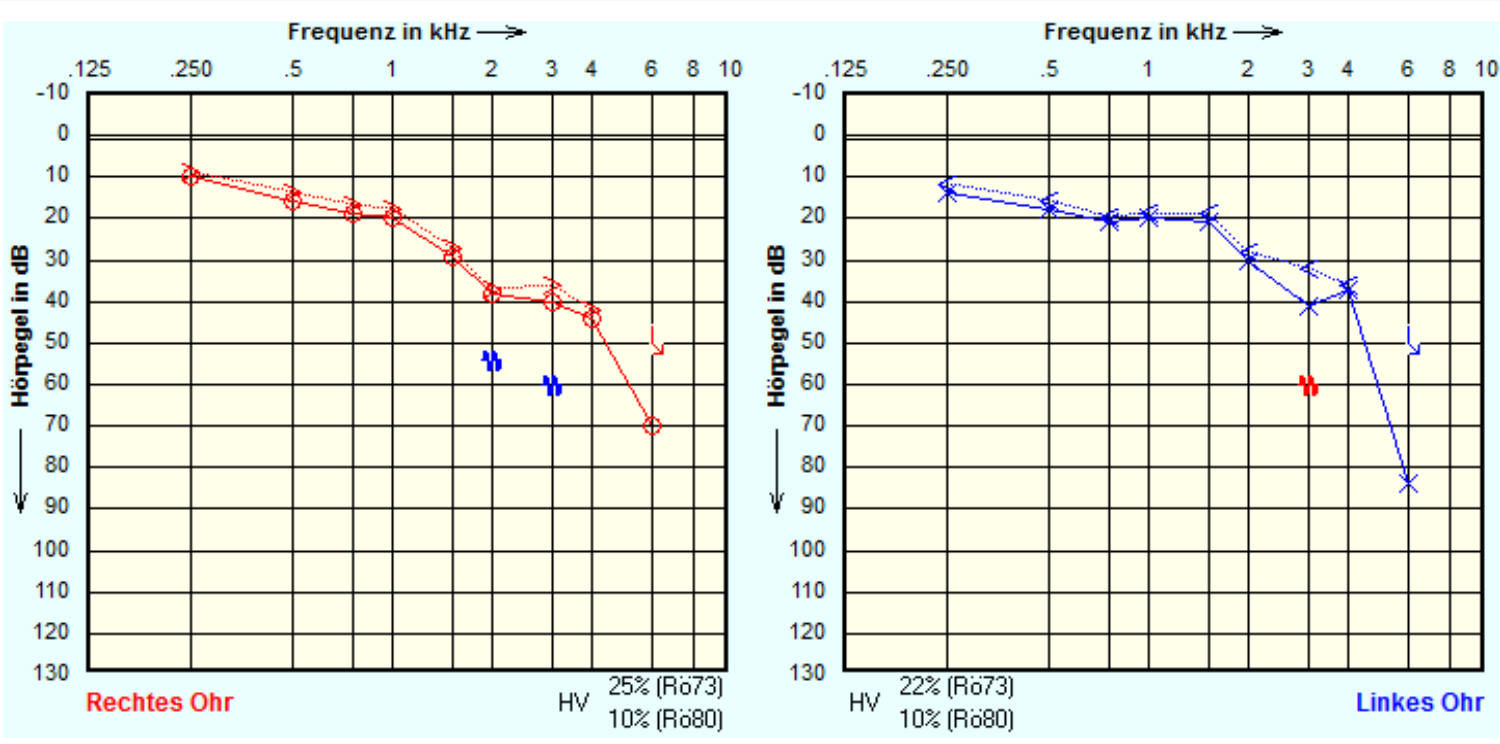

Figure 2: Audiogram on third day of therapy. 
According to $O$ Michel, et al. after presentation of 9 cases of patients with sudden sensorineural hearing loss following myelography, lumbar puncture or spinal anaesthesia, it seems probable that this rare complication arises only in patients with a wholly or partially patent cochlear aqueduct, allowing loss of perilymphatic fluid into the cerebrospinal space. A decrease in perilymphatic pressure is transmitted into the cochlea inducing a compensatory expansion of the endolymphatic compartment and causes a displacement of the hair cells in the basement membrane. This hydrops leads typically to low frequencies SSNHL due to compensatory expansion of the endolympatic space.. In this cohort were 8 of the 9 patients in the lower frequencies affected and six out of nine patients were bilateral affected. Not all of the hearing losses were fully reversible (only six out of nine cases) [9].

Pogodzinski, et al. described a case of a man who presented with a subjective and objective low-frequency SSNHL in addition to headache 4-days after he had undergone a dural puncture. He was treated with a standard epidural blood patch and immediately after treatment his hearing was improved and his headache resolved [10]. Crawford, et al. reported a case of transient acute on chronic hearing loss after lumbar puncture was performed to investigate raised intracranial pressure [11]. Satzer, et al. reported substantial evidence that CSF circulation and pressure abnormalities can produce auditory dysfunction in patients with hydrocephalus or CSF shunt placement. In their hydrodynamic theory they propose an intimate relationship between CSF and inner ear fluids, permitting relative endolymphatic or perilymphatic hydrops in the setting of CSF pressure disturbances. CSF communicates with perilymph, and CSF pressure changes are known to produce parallel perilymphatic pressure changes. Patients with hydrocephalus should be monitored for hearing loss. Patients with shunted hydrocephalus should prompt further evaluation and possibly adjustment of shunt settings [12].

Therapy of a SSNHL will be controversial in the literature discussed. Sara et al. reviewed articles concerning a bilateral SSNHL. In those cases of patients with bilateral SSNHL are therapy effects following a treatment either unchanged or progressive in $45,6 \%$ of cases. In $21,4 \%$ of patients was reported a complete remission of the symptoms and in 26, 2 $\%$ was only a partial remission documented. The application of steroids, were utilised, were most effective in cases of partial resolution in hearing outcome (36\%) than in cases with complete hear impairment (16\%). Treating cases of bilateral SSNHL with steroids resulting from vascular, toxic, immune or infectious diseases, seems to be more profitable for the patient in achieving hearing restoration [8]. Our experience aligned to current guidelines and we suggest steroids for the treatment.
Hearing loss, aural fullness, tinnitus or ear pressure will be mentioned by the patients from physicians as a temporary, isolated or rare complication after a LP. Our patient's case as well as case reports in the literature revealed variable implications that may result after CSF loss. Patients may experience from secondary temporary hearing deficits up to deafness. A clinical history to evaluate the hearing status of the patient or to select between high-risk patients, such as patients with a Menière syndrome, is therefore recommended. Medical staff and physicians should be aware of this rare complication [13].

\section{References}

1. National Institute on Deafness and Other Communication Disorders (2018) NIDCD fact sheet: Sudden deafness. US Department of Health and Human Services, Washington, DC, USA.

2. Chandrasekhar SS, Tsai Do BS, Schwartz SR, et al. (2019) Clinical practice guideline: Sudden hearing loss (Update). Otolaryngol Head Neck Surg 161: S1-S45.

3. (2017) QuickStats: Percentage of adults aged $\geq 18$ years with any hearing loss, by state - National Health Interview Survey, 20142016. MMWR Morb Mortal Wkly Rep 66: 1389.

4. Chung JH, Cho SH, Jeong JH, et al. (2015) Multivariate analysis of prognostic factors for idiopathic sudden sensorineural hearing loss in children. Laryngoscope 125: 2209-2215.

5. Mallepally AR, Rustagi T, Chhabra HS, et al. (2019) Sensorineural deafness after spine surgery: Case series and literature review. World Neurosurg 131: e482-e485.

6. Alwan M, Hurtado G (2019) Unilateral permanent sudden sensorineural hearing loss after spina-epidural anaesthesia for caesarean section: A case report. J Surg Case Rep 2019: 316.

7. Vilhena D, Pereira L, Duarte D, et al. (2016) Sudden sensorineural hearing loss after orthopedic surgery under combined spinal and epidural anesthesia. Case Rep Otolaryngol 2016: 4295601.

8. Sara SA, Teh BM, Friedland P (2014) Bilateral sudden sensorineural hearing loss: Review. J Laryngol Otol 128: S8-S15.

9. Michel O, Brusis T (1992) Hearing loss as a sequel of lumbar puncture. Ann Otol Rhinol Laryngol 101: 390-394.

10. Pogodzinski MS, Shallop JK, Sprung J, et al. (2008) Hearing loss and cerebrospinal fluid pressure: Case report and review of the literature. Ear Nose Throat J 87: 144-147.

11. Crawford A, McGrath NM (2010) Hearing loss after lumbar puncture. J Clin Neurosci 17: 149-152.

12. Satzer D, Guillaume DJ (2016) Hearing loss in hydrocephalus: A review, with focus on mechanisms. Neurosurg Rev 39: 13-24.

13. Irvin SM (2002) Sensorineural hearing loss after select procedures. J Perianesth Nurs 17: 89-98.

DOI: $10.36959 / 605 / 561$

Copyright: (c) 2021 Bezas V et al. This is an open-access article distributed under the terms of the Creative Commons Attribution License, which permits unrestricted use, distribution, and reproduction in any medium, provided the original author and source are credited.

SCHOLARS.DIRECT 